\title{
Solar wind elemental abundances related to the Sun's open magnetic flux
}

\author{
X. Wang ${ }^{1}$, B. Klecker ${ }^{2}$, and P. Wurz ${ }^{3}$ \\ 1 Key Laboratory of Solar Activity, The National Astronomical Observatories, CAS, 20A Datun Road, Chaoyang District, \\ 100012 Beijing, PR China \\ e-mail: xywang@nao.cas.cn \\ 2 Max-Planck-Institut für extraterrestrische, Physik, 85740 Garching, Germany \\ e-mail: berndt.klecker@mpe.mpg.de \\ 3 Physikalisches Institut der Universität Bern, 3012 Bern, Switzerland \\ e-mail: peter.wurz@space.unibe.ch
}

Received 19 November 2008 / Accepted 23 June 2009

ABSTRACT

\begin{abstract}
Context. We present an investigation of the effects of solar magnetic field on the elemental abundance ratios of heavy ions in the solar wind.

Aims. We compare the elemental abundance ratios $\mathrm{Fe} / \mathrm{O}, \mathrm{Si} / \mathrm{O}, \mathrm{Mg} / \mathrm{O}, \mathrm{Ne} / \mathrm{O}$, and $\mathrm{C} / \mathrm{O}$ over the $23 \mathrm{rd}$ solar cycle, attention being given to the varying fraction of open magnetic flux on the visible side of the solar disc.

Methods. We use ACE solar wind data, the current sheet source surface (CSSS) model of the corona, and SoHO/MDI data to relate solar wind compositional properties with the fraction of open magnetic flux on the Sun. We include both a quasi-stationary and intermittent solar wind.

Results. For the six investigated solar wind speed ranges, the elemental composition shows a similar FIP-bias of the quasi-stationary solar wind composition with respect to the photosphere but with three plateaus separating low-, mid-, and high-FIP elements. The average enrichment factor for the low-FIP element group (Mg, Fe, Si) with respect to the photospheric values is between 1.67 and 2.47 when the fraction of open magnetic flux on the visible side of the solar disc is above 14\%, and it ranges from 2.78 to 4.15 when this fraction is less than 7\%. They are close to the enrichment of low-FIP elements with respect to the photospheric values in quasistationary fast- and slow- solar wind, which implies similar fractionation mechanisms for the stationary solar wind and the intermittent solar wind. In the low alpha $(<7 \%)$ case (alpha is the fraction of open magnetic flux on the visible side of the solar disc), the enrichment of heavy elements for the fast solar wind is well correlated with the high fraction of CMEs. In addition, both the charge state ratios and the elemental abundance ratios exhibit a lower dependence on alpha in a slow solar wind than in a fast solar wind. The dependence of the charge state ratios on alpha is correlated with FIP, which implies that solar magnetic effects play a non-negligible role in the elemental fractionation of the solar wind. We suggest that lower hybrid waves play an important role in the possible linkage between solar magnetic field and elemental fractionation.
\end{abstract}

Key words. Sun: abundances - Sun: activity - Sun: solar wind - interplanetary medium

\section{Introduction}

The magnetic field of the solar surface and atmosphere includes two components: open magnetic flux, which opens into the heliosphere to form the heliospheric magnetic field; and closed magnetic flux, in the form of loops attached to both ends of the solar surface. The open flux controls many of the important processes in the solar corona with the release of solar wind into the interplanetary space being one of them. At higher particle energies, Reames (1999), for example, argues that the interaction of the loop with the open flux is essential to an impulsive solar particle event, triggering both the redistribution of the magnetic field in the loop and the escape of energetic particles. A model was developed for the acceleration of the solar wind, in which the source of energy and plasma is the reconnection of open fields lines with closed magnetic loops, and the subsequent displacement of the open field lines and release of material from the loop (Fisk et al. 2003; Fisk \& Zurbuchen 2006). This model places several important constraints on the mass and energy flux, and the final speed of the solar wind, and relates these quantities to basic solar parameters such as the emergence of new magnetic flux on the Sun (Fisk 2003). The interaction and reconnection between open flux and coronal loops distributes open flux broadly over the Sun (Fisk et al. 2003; Fisk \& Zurbuchen 2006). The solar wind coming from open flux regions in or near active regions has properties that differ from those of than the solar wind from the traditional coronal holes (Liewer et al. 2004). It is thus important to differentiate originating in open flux and coronal holes because the definition of a coronal hole is an observational one (Levine 1982).

It is well established that the relative abundances of elements in the solar corona, solar wind, and solar energetic particles are almost similar (Meyer 1985). However, compared to the photosphere, the particle populations show a fractionation that is organized by the first ionization potential. From composition measurements, it is known for the solar wind plasma and energetic particles that the elements with a low FIP (i.e., FIP $<10 \mathrm{eV}$ ) exhibit a systematical enrichment with respect to their photospheric abundances, by a factor of between 2 and 4 for slow solar wind, and a factor of between 1.5 and 2 for fast 
solar wind. The FIP effect is largely discussed in the literature, and there exist several models that give account of it, for example Marsch et al. (1995), Arge \& Mullan (1998), Schwadron et al. (1999), and Laming (2004). These models differ in terms of the underlying physical processes (Hénoux 1998), and at present there is no consensus about the mechanism responsible for the FIP fractionation in the solar wind. Moreover, when looking in detail at the various particle populations do show some differences, as demonstrated for $\mathrm{Ca}$ in the solar wind (Wurz et al. 2003a). In particular, energetic particles often show severe fractionation in their elemental composition. On the other hand, low-speed CMEs are the most difficult to identify within the ambient low-speed solar wind (Reisenfeld et al. 2003). In many aspects, CME-related flow reflects most closely the lowspeed solar wind, except for the general enhancement of He. It is still an open question whether the CME-related solar wind needs to be considered as an independent type of flow, or whether a low-speed solar wind reflects a composition, produced by a multitude of small-scale CMEs, that dissolve in the inner corona, while only the large-scale CMEs survive out to spacecraft observations and remain identifiable as independent events (Bochsler 2007). However, the elemental composition during the passage of the January 6, $1997 \mathrm{CME}$ was found to differ from the interstream and coronal hole regions before and after it with a massdependent element fractionation (Wurz et al. 1998). In a study of CME plasma composition in the velocity range from 390 to $520 \mathrm{~km} \mathrm{~s}^{-1}$, a strong deviation in the composition of heavy ions with respect to slow solar wind was found for each CME (Wurz et al. 2003b). Schwadron et al. (1999) demonstrated that wave heating can account for both a mass-independent fractionation and a bias of low-FIP elements on active region loops, but not on continuously open field lines. Their model placed several constraints on the rate at which waves isotropize and thereby heat the species distributions, e.g., the spatial scale of the discontinuity must be smaller than the gyroradius of protons. In examining the physical processes that can account for the mass-dependent element fractionation, we focus on the waves associated with anisotropic ions that would act more significantly on heavy ions than on protons. We include both a quasi-stationary and intermittent solar wind and pay particular attention to the abundance variations associated with the solar magnetic effects.

The evolution in the open magnetic flux on the solar surface reflects the changing properties of the solar activity. The solar wind associated with the distribution of the open flux is also expected to provide the information about the solar activity. Significant abundance enhancements are observed in the solar corona in open magnetic field structures such as polar plumes (Feldman 1992; Sheeley 1996) and in active regions surrounding a sunspot with diverging magnetic field lines (Doschek 1983). Based on models with impulsive events, a significant fraction of heavy elements that reside in the actively flaring flux rope is energized, and the resonant interaction operates mainly on heavy elements with charge states that increase systematically with energy (Möbius et al. 2003; Klecker et al. 2006; Kartavykh et al. 2007). We are therefore motivated to explore the magnetic effects on the abundance variations of heavy ions in the solar wind plasma.

\section{Data analysis}

Photospheric magnetic fields are extrapolated into a global heliospheric field by using the current sheet source surface (CSSS) model (Zhao \& Hoeksema 1995). This model uses Bogdan and Low's solution (Bogdan \& Low 1986) for a magnetostatic equilibrium to calculate the effect of large-scale horizontal currents flowing in the inner corona and, by introducing the cusp surface and the source surface, uses Schatten's technique (Schatten 1971) to calculate the effects of the coronal and heliospheric current sheets and volume currents. These currents maintain the total pressure balance between regions of high and low plasma density. To model the effects of volume and sheet currents on the coronal magnetic field, the solar atmosphere is divided into three parts, separated by two spherical surfaces, which are the cusp surface and the source surface (see Fig. 1 of Zhao \& Hoeksema 1995). The inner sphere, called the cusp surface, is located approximately at the height of the cusp points of coronal streamers. Above the cusp surface, the coronal magnetic field is open everywhere. The outer sphere, called the source surface, is located closed to the reference height identified in Parker's model above which the radially directed solar wind totally controls the magnetic field (Parker 1958). The cusp point is not easily defined and probably varies with position. However, the estimates of the height of cusp points from different experiments range from below 1.5 solar radii to above 3 solar radii (Zhao \& Hoeksema 1995). For instance, typical coronal streamers in the K corona are approximately radial structures extending beyond 1.5-2.0 solar radii. With different sensitivitues, the cusp points of helmet streamers would range at different heights, e.g., they range at 2-3 solar radii in eclipse images (e.g. Koutchmy 1977 ) and they range at $1.15-1.5$ solar radii in soft X-rays by the Yohkoh spacecraft (Zhao \& Hoeksema 1995). This implies that the interactions between the open and closed field lines can happen over a wide height range.

There are two essential advantages of the CSSS model over the potential field-source surface (PFSS) model: first, the field lines are open but not necessarily radial on the cusp surface, which includes the effects of streamer current sheets; second, the source surface in the CSSS model is placed close to the Alfvén critical point across which the solar wind speed changes from sub-Alfvénic to super-Alfvénic. In-situ observations of the heliospheric magnetic field should be compared with the magnetic neutral line close to the Alfvén critical point. The radial component of the heliospheric magnetic field, as detected by Ulysses, is latitude-independent (Smith \& Balogh 1995), and can be taken as uniform on a spherical surface above 5 solar radii (Suess \& Smith 1996). However, the magnetic field distribution on the source surface obtained using the PFSS model is not uniform, in disagree with the Ulysses observations of the heliospheric magnetic field (Poduval \& Zhao 2004). In addition, the CSSS model shows a superior prediction of solar wind and interplanetary magnetic field (IMF) polarity and intensity measured close to the Earth's orbit than the potential field-source surface (PFSS) model. The correlation coefficient between the observed interplanetary magnetic field and the calculated 27-day averages is 0.89 for the CSSS model, which is superior to that of the PFSS model, where the average is 0.77 (Zhao \& Hoeksema 1995). To obtain a uniform magnetic field on the source surface, we set the source surface to be at 15 solar radii (Zhao et al. 2002) and the optimum cusp surface was determined by matching trial calculations of Carrington rotation-averaged open magnetic fluxes with in-situ solar wind speed.

The global potential field extrapolation is a good approximation of the large-scale magnetic field structures in the corona. Apart from in very simple magnetic field configurations, the insitu measured solar wind would be associated with material released from several related sources on the solar surface (because of their complex magnetic reconections). So it is not easy to set a small local relevant source region by an identical criterion. 
Table 1. Distribution of the number of the 2-h points in the five alpha bins and six velocity bins.

\begin{tabular}{ccccccc}
\hline \hline alpha & $250<v<350$ & $350<v<450$ & $450<v<550$ & $550<v<650$ & $650<v<750$ & $750<v<850$ \\
\hline$[13,35]$ & 765 & 1745 & 1456 & 1214 & 885 & 417 \\
{$[11,13]$} & 774 & 1898 & 1511 & 988 & 441 & 108 \\
{$[9,11]$} & 1079 & 3233 & 2186 & 1247 & 438 & 84 \\
{$[7,9]$} & 1312 & 3838 & 2669 & 1048 & 443 & 62 \\
{$[0,7]$} & 1674 & 3547 & 1751 & 621 & 181 & 49 \\
\hline
\end{tabular}

Our interest is in the effects of the magnetic field on the solar wind, not only the relevant magnetic configuration of source region. Liu \& Hayashi (2006) ascertained the role of the configurations of the background magnetic field in determining coronal disturbance propagation. They found that when an active region is within an open flux area, the related CMEs are faster than those without open flux (Liu \& Hayashi 2006). Chen (1996) also demonstrated the influence of the overlying field on the motion of the flux rope that had erupted because of the injection of the poloidal flux into the system. Chen found that when the flux rope moved upward after eruption, the major forces withholding its motion were the Lorentz force from the toroidal current, the background field, and the gravity (see Fig. 7 of Chen 1996). Based on these results, we conjecture that the role of the background magnetic field configurations on the local source should not be negligible in considering the effects of solar magnetic field on the solar wind. Therefore, in the following mapping technique, we associate the solar wind with the fraction of open magnetic flux across the entire visible solar disc.

SoHO/MDI daily magnetic field synoptic data are used to obtain the daily fraction of open magnetic flux on the visible side of the solar disc (Fig. 1a) during the 23rd solar cycle (alpha $=$ opnf/(opnf+clsf), where opnf (clsf) indicates open (closed) flux on the visible side of the solar disc). Near sunspot minimum, open magnetic flux occupies more than $20 \%$ of the solar surface and is concentrated across the large polar holes. With the disappearance of the large polar holes at sunspot maximum, the total area occupied by open magnetic fields decreases to only $5 \%$ of the solar surface. In a correlation study, we found that the open magnetic flux and sunspot number are anticorrelated with a correlation coefficient of 0.46 (see Fig. 1b). This implies that the fraction of the open magnetic flux in the photosphere can be used to describe the solar activity. The fraction of the open magnetic flux may leave some indications of the properties of the solar wind.

The observed solar wind is traced back to the source surface in the corona along the Archimedian spiral assuming little radial acceleration (constant speed) and pure radial flow, neglecting interaction between fast and slow solar wind streams. That is, the heliographic latitude at the source surface is the same as that of in-situ observed point. The Carrington longitude at the source surface is shifted to the west according to the daily values of in-situ observed solar wind speed (Neugebauer et al. 2002). Daily average solar wind data as measured by ACE/SWEPAM are used to infer the longitude shift. Once the shifted longitude is obtained, we derive both the time of day when the solar wind emerged from the source surface and the daily fraction of open magnetic flux on the front side of the photosphere for that day. This inverse mapping technique enables us to associate the solar wind elemental composition with the fraction of open magnetic flux on the visible side of the solar disc. We do not use the inverse relation between flux tube expansion factor and solar wind speed, because the solar wind will be tracked back to a region bounded within a narrow range of longitude that depends
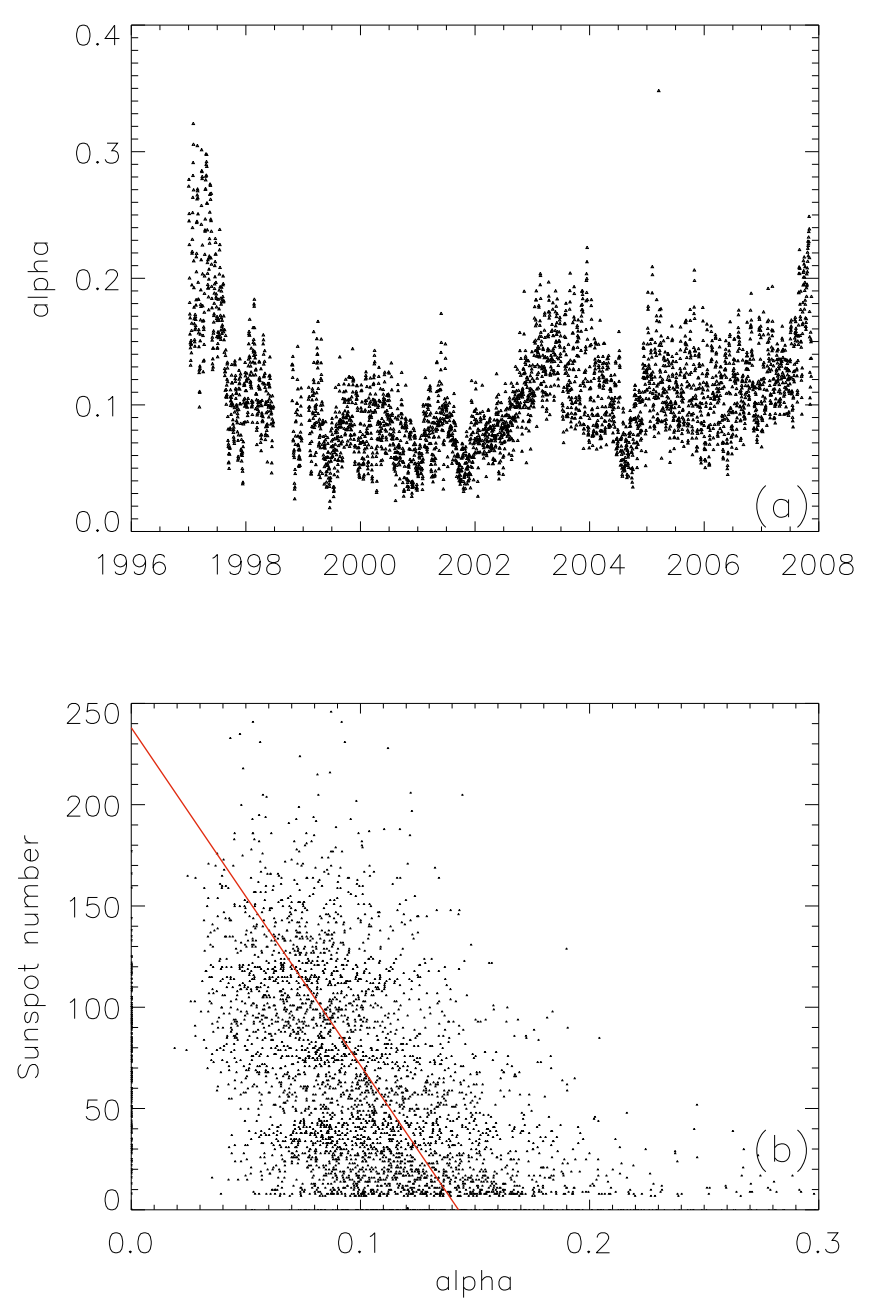

Fig. 1. a) The fraction of the open magnetic flux on the visible side of the solar disc (alpha is the fraction of open magnetic flux) as a function of time during solar cycle 23rd. b) The solid line shows the correlation between sunspot number and open magnetic flux with a correlation coefficient -0.46 .

sensitively on the mapping speed used, but rather we consider the influence of the background magnetic field on the properties of solar wind. We note that the solar wind speed profile obtained at $1 \mathrm{AU}$ is the result of the interaction between solar wind parcels of different speeds as they propagate outwards and that the stream-stream interactions are inevitable. Our constant speed assumption would inevitably introduce a longitude shift error and possibly cause an incorrect mapping of the solar wind with solar magnetic field. However, we use the daily synoptic MDI data for the mapping that slightly weakens the influence of the longitude shift error. For instance, an error of less than 13 degree would not change the mapping result. 

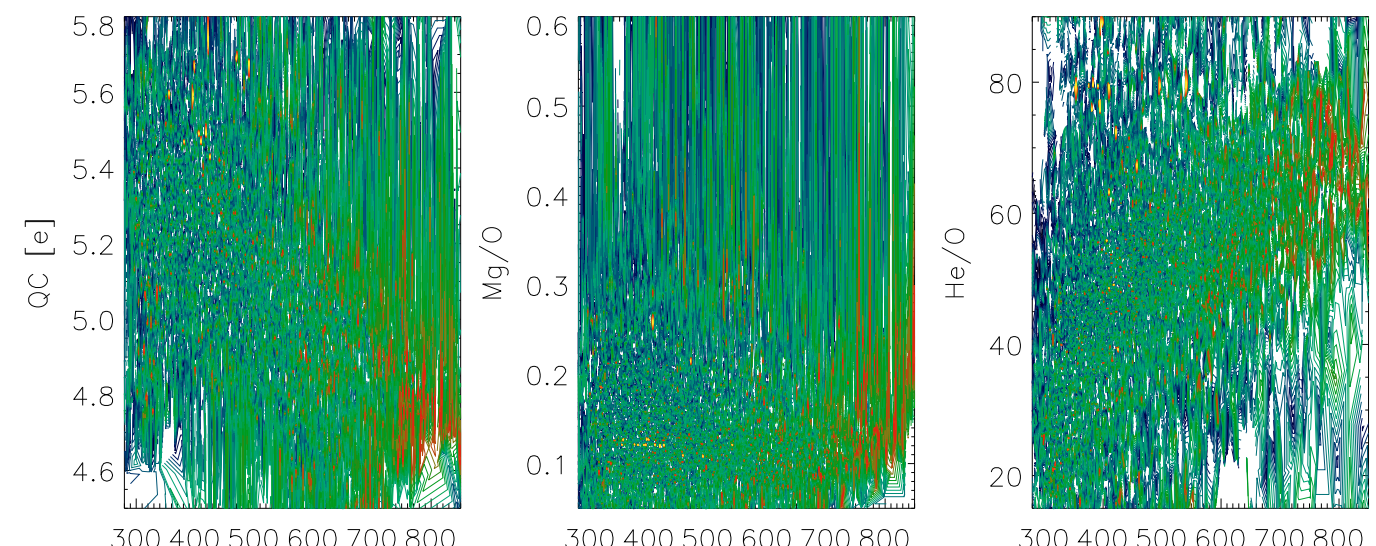

alpha
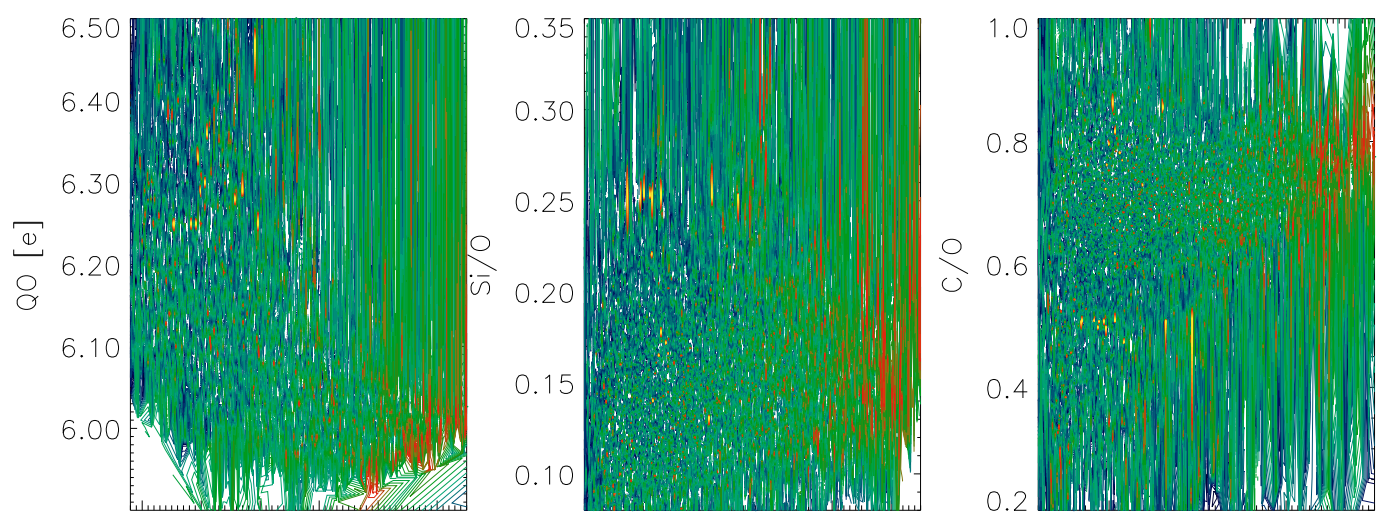

$23 \%$

300400500600700800

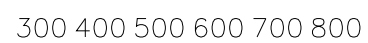

300400500600700800

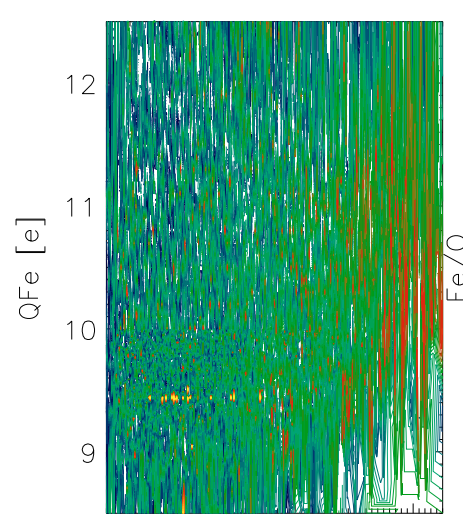

300400500600700800

$$
\operatorname{Vsw}(\mathrm{km} / \mathrm{s})
$$

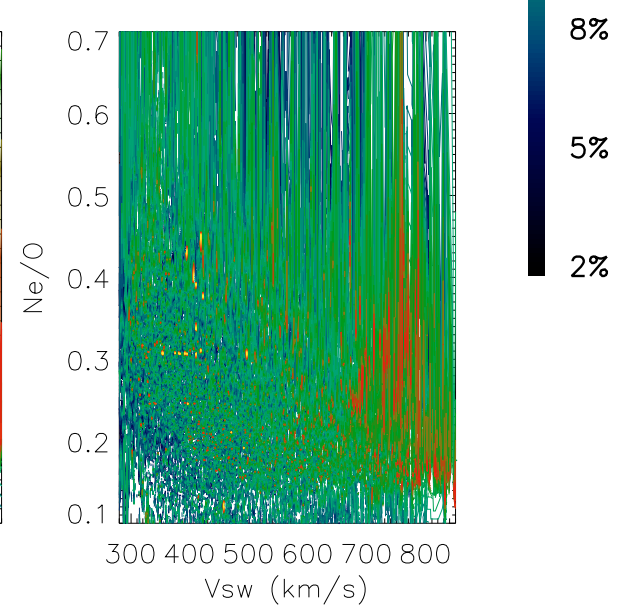

$8 \%$

$5 \%$

$2 \%$

Fig. 2. 3D plots for the charge states and elemental abundance ratios versus solar wind velocity with different alpha (alpha represents the fraction of the open magnetic flux on the visible side of the solar disc). QFe, QO, and QC represent the charge states of iron, oxygen, and carbon, respectively. Each point corresponds to a two-hour average, and the survey covers the ACE mission from DOY 36, 1998 to DOY $110,2007$.

\section{Results}

We analyzed the elemental abundance ratios $\mathrm{Fe} / \mathrm{O}, \mathrm{Mg} / \mathrm{O}, \mathrm{Si} / \mathrm{O}$, $\mathrm{Ne} / \mathrm{O}, \mathrm{C} / \mathrm{O}$, and $\mathrm{He} / \mathrm{O}$ as measured by $\mathrm{ACE} / \mathrm{SWICS}$, with attention given to the fraction of the open magnetic flux. In Fig. 2, we compiled the 3D plots for the charge states and elemental abundances versus solar wind speed for different alpha value. Each data point corresponds to a two-hour average. This survey covers the data from DOY 36, 1998 to DOY 110, 2007 of the ACE mission. The quality flags that we used are quality flag $=0$ and 1 .

Mean abundance ratios and the charge state ratios relative to oxygen for the six solar wind speed bins are compiled in Fig. 3. Considering the ratios in Fig. 3, the systematic error would be a second order effect, the indicated uncertainties being only the statistical $1 \sigma$ error. For the low-FIP elements Fe and Si, we find that the charge state ratios $\left(Q_{\mathrm{O}} / Q_{\mathrm{Fe}}, Q_{\mathrm{O}} / Q_{\mathrm{Si}}\right)$ in the low alpha bin $($ alpha $<7 \%)$ are lower than the ratios in the higher alpha bins when the solar wind velocity is between $550 \mathrm{~km} \mathrm{~s}^{-1}$ and $750 \mathrm{~km} \mathrm{~s}^{-1}$, and they tend to decrease with solar wind speed. Although this dependence is not obvious in another low-FIP element $\mathrm{Mg}, Q_{\mathrm{O}} / Q_{\mathrm{Mg}}$ still exhibits some decrease in the low alpha bin (alpha $<7 \%)$ when the solar wind velocity is between $650 \mathrm{~km} \mathrm{~s}^{-1}$ and $750 \mathrm{~km} \mathrm{~s}^{-1}$. For the high-FIP element neon, $Q_{\mathrm{O}} / Q_{\mathrm{Ne}}$ in the low alpha bin (alpha $<7 \%$ ) is found to be higher than the ratios in the higher alpha bins when the solar wind velocity is between $450 \mathrm{~km} \mathrm{~s}^{-1}$ and $650 \mathrm{~km} \mathrm{~s}^{-1}$. For the mid-FIP 
$\mathrm{X}$. Wang et al.: Elemental abundances related to open magnetic flux
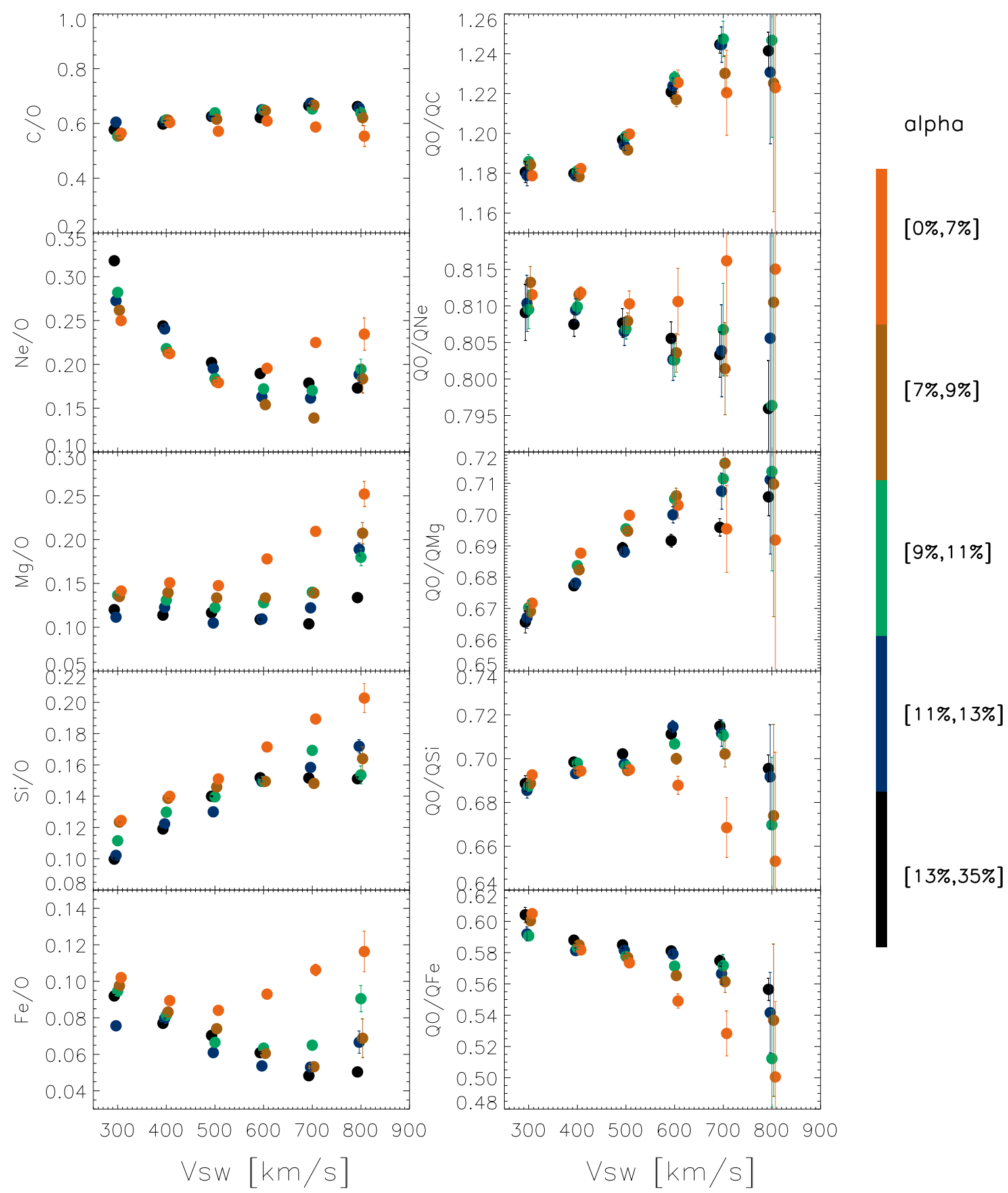

Fig. 3. Elemental abundance ratios and charge state ratios (relative to oxygen) versus solar wind speed with different alpha. The uncertainties in the plot represent the statistical $1 \sigma$ error.

element carbon, $Q_{\mathrm{O}} / Q_{\mathrm{C}}$ does not show any prominent difference between different alpha bins. In other words, the variation of charge state ratios with alpha seems to be correlated with FIP. To highlight this correlation more clearly, we compile the variation in charge state ratios into different alpha bins as a function of FIP for the velocity bin $650 \mathrm{~km} \mathrm{~s}^{-1}<V s w<750 \mathrm{~km} \mathrm{~s}^{-1}$ in Fig. 4, where CR indicates the ratio $Q_{\mathrm{O}} / Q_{\mathrm{X}}$, it is clear that CR for the alpha $<7 \%$ bin to CR in $7 \%<$ alpha $<9 \%$ bin exhibits FIP dependence. We fit this dependence with the function $y=0.847^{*} x^{* *} 0.06$, which is indicated by a red line. This not only indicates the non-negligible role of the configuration of background magnetic field in determing the charge states, considering the wave-particle interactions, it also suggests that solar magnetic effects play an important role in the elemental fractionation of the solar wind.

We note that both the charge state ratios and the abundance ratios in the slow solar wind do not differ significantly between different alpha bins. In the low alpha bin $(<7 \%)$, the overall trend of the abundance ratios in the fast solar wind is clear: the abundance ratios rise with the solar wind velocity when the mass is above that of oxygen. When including a $10 \%$ to $20 \%$ measurement error for the ACE abundance data, the variation in $\mathrm{C} / \mathrm{O}$ with alpha is lost and leads to a constant value of $\mathrm{C} / \mathrm{O}$ for all solar wind speeds. The ratio Fe/O basically decreases with solar wind speed when alpha is relative high, which agrees with the results of previous work (e.g., Wurz et al. 1999; 


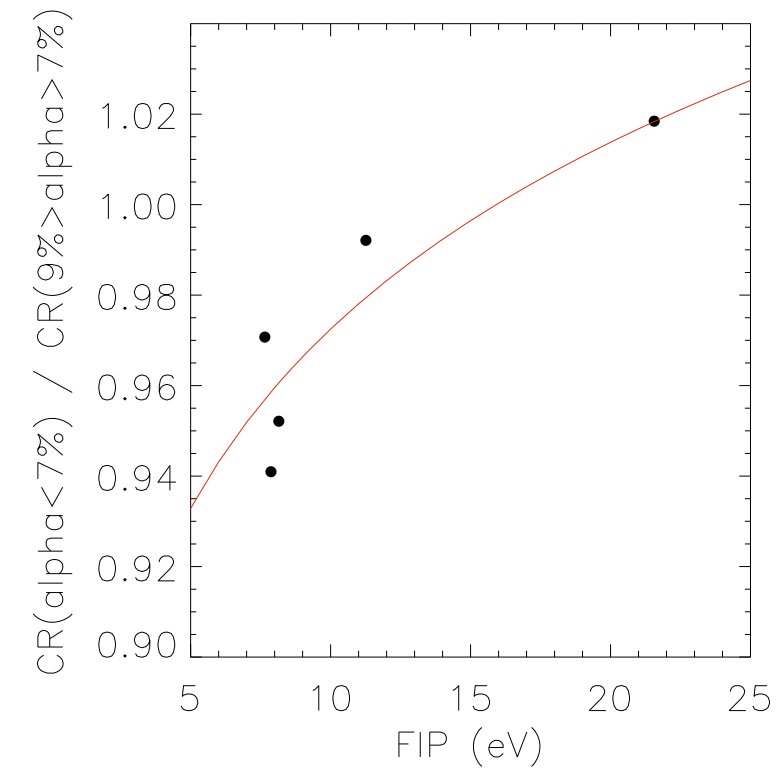

Fig. 4. Variation in charge state ratios in different alpha bins as a function of FIP for the velocity bin $650 \mathrm{~km} \mathrm{~s}^{-1}<V s w<750 \mathrm{~km} \mathrm{~s}^{-1}$. CR indicates the ratio $Q_{\mathrm{O}} / Q_{\mathrm{X}}$. The axis of ordinate is the ratio of $\mathrm{CR}$ in alpha $<7 \%$ bin to CR in $7 \%<$ alpha $<9 \%$ bin, which increases from 0.94 to 1.02 as FIP is increasing. The red line shows a least squares fit to the data.

Aellig et al. 1999). The difference occurs as the parameter alpha decreases. As we know, CMEs, which may be associated with high solar activity, can increase by a large amount in the abundances of heavy elements, especially iron. These increases are in addition to the enrichments of the FIP effect, where a factor 4 to 10 in enrichment for iron in the CME plasma is possible in individual events (Wurz et al. 1998, 2003b). We show the distribution of the three types of solar wind and the fraction of CMEs in the five alpha bins and six velocity bins in Fig. 5. When alpha is below $7 \%$, the fraction of the CMEs increases from $1 \%$ to $71 \%$ as the solar wind speed increase from $300 \mathrm{~km} \mathrm{~s}^{-1}$ to $800 \mathrm{~km} \mathrm{~s}^{-1}$. If we attempt to link statistically the high solar wind speeds with the high fraction of CMEs during active times, we can explain the enrichment of heavy ions for the fast solar wind in the low alpha bin. Statistically, the correlation between solar activity and CMEs is established well (e.g., Hildner et al. 1976; Kahler 1992; Wang et al. 1998; Zhou et al. 2003; Gopalswamy et al. 2003; Yashiro et al. 2004).

However, $\mathrm{Si} / \mathrm{O}$ and $\mathrm{Mg} / \mathrm{O}$ behave differently from $\mathrm{Fe} / \mathrm{O}$ because their abundance ratios do not decrease with solar wind speed even for the high alpha bins. We note that this is a statistical analysis of samples including all types of solar wind, and might differ from the results of a more limited analysis that is typical of abundance studies. The role of the magnetic effect cannot be neglected in considering this difference. Is it possible that the magnetic effect plays a more prominent role for $\mathrm{Si}$ and $\mathrm{Mg}$ than for Fe in the high alpha bins? One of our previous statistical study demonstrates that iron displays higher charge states in a fast wind than in a slow wind (Wang et al. 2008), but for silicon and magnesium, the mean charge states basically decrease with the velocity ( $V s w<750 \mathrm{~km} \mathrm{~s}^{-1}$ ) in the high alpha bins (Wang et al. 2008). If the low abundance ratio of $\mathrm{Fe} / \mathrm{O}$ in the fast wind when alpha is above $7 \%$ can be partly attributed to the high charge states of $\mathrm{Fe}$ in fast wind, we can understand the lack of decrease in $\mathrm{Si} / \mathrm{O}$ and $\mathrm{Mg} / \mathrm{O}$ for fast wind in the high alpha bins (more power resonating with $\mathrm{Mg}$ and $\mathrm{Si}$ than with $\mathrm{O}$ because

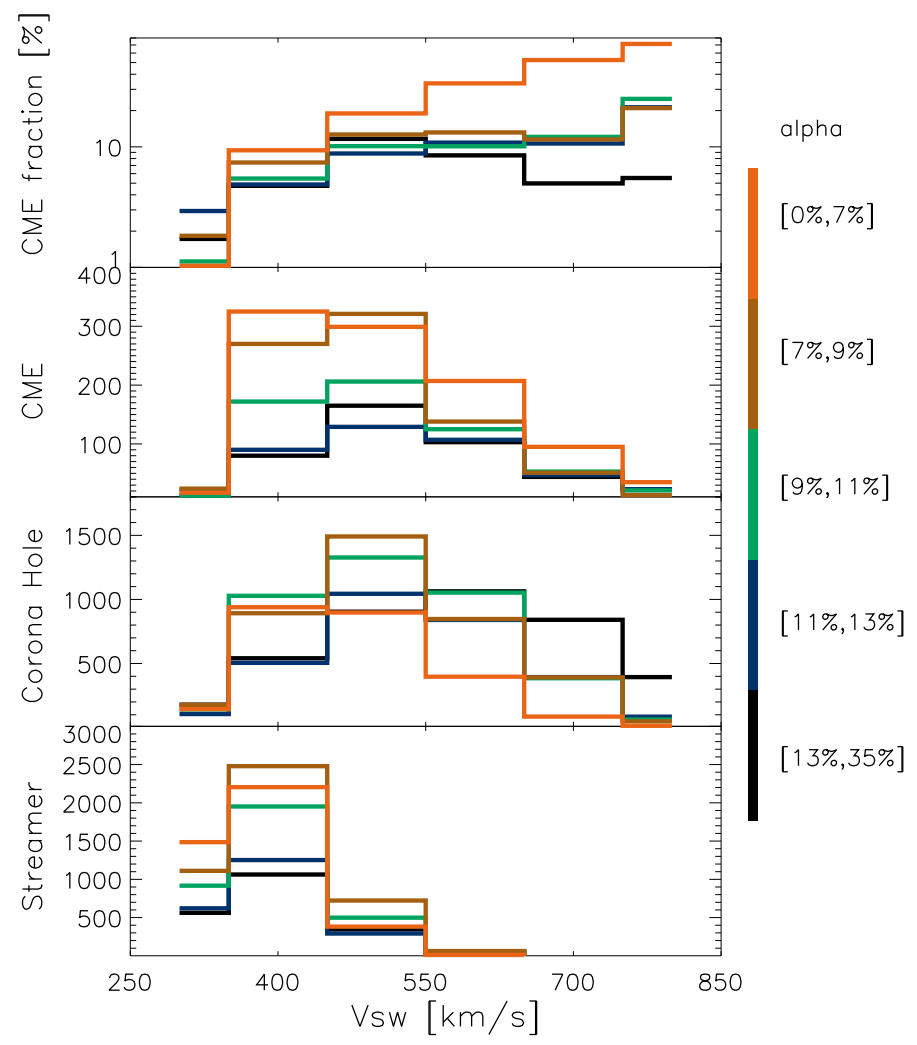

Fig. 5. Distribution of the three types of solar wind and the fraction of CMEs in the five alpha bins and six velocity bins.

of their lower charge states). In other words, because of waveparticle interactions, the increased ratio of charge states $\left(Q_{\mathrm{O}} / Q_{\mathrm{Si}}\right.$ or $\left.Q_{\mathrm{O}} / Q_{\mathrm{Mg}}\right)$ would result in an abundance ratio $(\mathrm{Si} / \mathrm{O}$ or $\mathrm{Mg} / \mathrm{O})$ that had not decreased in the fast solar wind.

The more prominent role of solar magnetic effects in fast solar wind may be caused by the higher electron temperature powered by the MHD waves at the relative high altitude in the corona, i.e., at 1.5-3 solar radii. Assuming that the fast solar wind is accelerated by ion cyclotron waves generated by the interaction and reconnection between open flux and small-scale closed loops at this height, ions are perpendicularly heated by the ion cyclotron waves and execute large gyro-orbits. Density gradients in the flow can then excite lower hybrid waves through which electrons can then be heated in a parallel direction (Laming \& Lepri 2007). Once heated by lower hybrid waves, the enhanced electron temperature would lead the elemental charge states to a higher level. However, this would be different for a slow solar wind because the slow solar wind is believed to accumulate in closed loops for hours to days before releasing into the heliosphere (e.g., Uzzo et al. 2003). The ions execute sufficient heating in the loops and become almost isotropic. The conditions for exciting lower hybrid waves might not be satisfied. Therefore, the charge states and the abundance ratios in slow and fast solar wind respond differently to the solar magnetic effects.

We also study helium, another high-FIP element. Helium does not show a trend similar to that of neon because He cannot be regarded as a minor ion in the solar wind plasma and its fractionation may be associated with the bulk solar wind acceleration (see discussion in Aellig et al. 2001; Kasper et al. 2007).

In Fig. 6, we present an overview of the measured solar wind abundances underlying different alpha. For comparison, we plotted the elemental abundances as a function of both FIP (right column) and mass (left column). The observed solar wind 


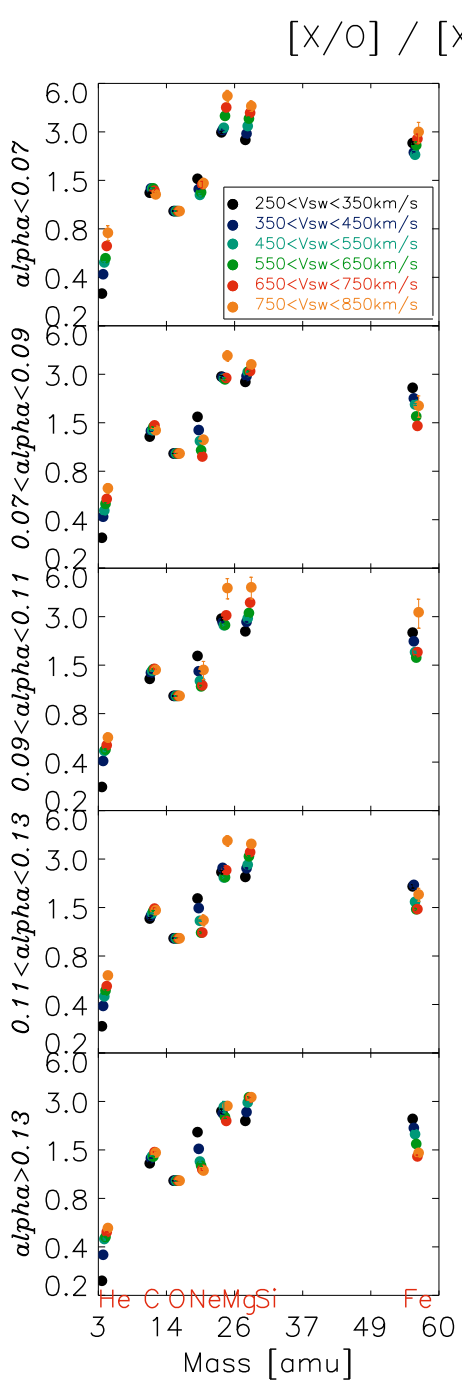

$[\mathrm{X} / \mathrm{O}]$ photosphere

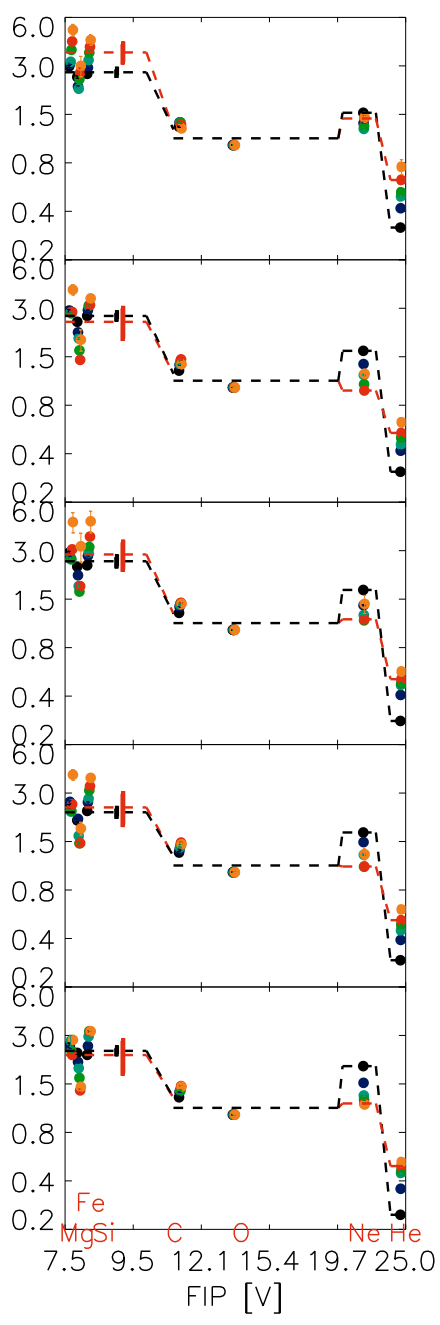

Fig. 6. Elemental abundance ratios (relative to oxygen) compared to photospheric ratios with different alpha, plotted as a function of FIP and mass. The FIP fractionation pattern results with basically three plateaus separated by a step at $10 \mathrm{eV}$ between the low-FIP elements (Mg, Fe, $\mathrm{Si}$ ) and mid-FIP elements ( $\mathrm{C}$ and $\mathrm{O}), \mathrm{Ne}$ is marked seperately from $\mathrm{C}$ and $\mathrm{O}$ because of its different behavior, another step is observed between the high-FIP element $\mathrm{Ne}$ and $\mathrm{He}$. The error bar indicated on the low-FIP plateau represents the standard deviation of the average height of the low-FIP element group (Fe, $\mathrm{Si}, \mathrm{Mg}$ ).

abundance ratios (relative to oxygen), compared to the photospheric abundance ratios, show the well-known FIP pattern. The abundance ratios as a function of FIP can be grouped into three plateaus: a low-FIP plateau for FIPs $<10 \mathrm{eV}$ with the elements $\mathrm{Mg}, \mathrm{Fe}$, and $\mathrm{Si}$ with largest enrichments, separated by a step at about $10 \mathrm{eV}$ until about $20 \mathrm{eV}$ for the relative high-FIP elements including $\mathrm{C}$ and $\mathrm{O}$ with enrichments around 1. We highlight the high-FIP element neon separately because it behaves differently from $\mathrm{C}$ and $\mathrm{O}$. The high-FIP element He often shows an abundance ratio of 0.5 (Aellig et al. 2001), i.e., it is depleted with respect to its photospheric abundance. The error bar marked on the low-FIP plateau represents the standard deviation of the average height of the low-FIP element group (Fe, $\mathrm{Si}, \mathrm{Mg}$ ).

In contrast to low-FIP (Mg, Fe, Si) and high-FIP elements $(\mathrm{Ne}, \mathrm{He})$, no obvious variations with solar wind speed are found for the two high-FIP elements carbon and oxygen (oxygen is used as a reference). Carbon is slightly enriched relative to $\mathrm{O}$, but this enrichment does not change significantly with the solar wind speed (see also Fig. 3). Therefore, the mid-FIP element plateau is plotted for a single plateau height that does not distinguish the fast wind from the slow wind. The average plateau height of the low-FIP element group (Fe, $\mathrm{Si}, \mathrm{Mg}$ ) shows visible enhancement in the fast solar wind when alpha is below 7\%, whereas it has no obvious variation in the slow solar wind for all alpha bins. This suggests that mechanisms enhancing the upward transport of low-FIP elements should work equally well in low-speed CMEs and the ambient low-speed solar wind. The average enrichment factor for the low-FIP group (e.g., $\mathrm{Mg}, \mathrm{Fe}, \mathrm{Si}$ ) relative to photospheric values is between 1.64 and 2.47 when alpha is above $14 \%$, and it ranges from 2.78 to 4.15 when alpha is below $7 \%$. For the high-FIP element $\mathrm{Ne}$, the plateau height for the low-speed solar wind is always higher than that for highspeed solar wind untill alpha is below 7\%, where they become close each other. The plateau heights for $\mathrm{Ne}$ between different velocity bins can vary by as much as a factor of two. This is in contrast to the result for a quasi-stationary solar wind, which is found to be constant and independent of solar wind type (e.g. von Steiger 1995; Geiss et al. 1994). However, it agrees with the spectroscopically determined $\mathrm{Ne} / \mathrm{O}$ ratios in active regions and flares (McKenzie \& Feldman 1992) and agrees quite closely with the photospheric value (Anders \& Grevesse 1989). Widing and Feldman (1992) demonstrated that the ratio $\mathrm{Mg} / \mathrm{Ne}$ measured for their spectroscopic observations can vary by as much as an order of magnitude, and its variation is correlated with the magnetic field morphology at the observed site. The largest $\mathrm{Mg} / \mathrm{Ne}$ values are found in polar plumes and diverging fields (Widing $\&$ Feldman 1992). Although their report triggered some debate about the effects of electron temperature or FIP (e.g., Doschek \& Laming 2000), since the dependence of the charge state ratios on alpha is correlated with FIP, it is quite possible that the effects of electron temperature and FIP effects complement each other in the elemental fractionation and, for example, by means of the redistribution of open magnetic flux on the solar surface, ion cyclotron waves are excited. Ions heated perpendicularly by ion cyclotron waves also excite lower hybrid waves by which electrons are heated (Laming \& Lepri 2007). An enhanced electron temperature also influences the charge states of ions. Through waveparticle interactions, the modification of charge states would finally be reflected in the elemental abundances.

\section{Summary}

We have presented the effects of a solar magnetic field on the charge states and elemental abundances of heavy ions in the solar wind. We have found that the enrichment of low-FIP elements with respect to the photospheric values is around 2 when the fraction of the open magnetic flux on the visible side of the solar disc is above $14 \%$, and rises to $2.78-4.15$ when the fraction of the open flux is below 7\%. These values are close to the enrichment levels of low-FIP elements with respect to the photospheric values in quasi-stationary fast- and slow- solar wind, which implies that the intrinsic mechanisms for the fractionation of stationary solar wind and intermittent solar wind might be similar. In the low alpha $(<7 \%)$ case, the enrichment of heavy elements for the fast solar wind is well correlated with the high fraction of CMEs. Both the charge state ratios and the elemental abundance ratios have a lower dependence on alpha (which represents the fraction of the open magnetic flux on the visible side of solar disc) in the slow solar wind than in the fast solar wind. The dependence of the charge state ratios on alpha is correlated with 
FIP, which implies that solar magnetic effects have an important role in the elemental fractionation of the solar wind. A possible linkage between solar magnetic field and elemental fractionation illustrates the important role of the lower hybrid waves.

Acknowledgements. The comments by the anonymous referee, which helped to improve this work, are greatly appreciated. We thank the ACE SWICS-SWIMS instrument team and $A C E$ S cience Center for providing the $A C E$ data. We thank $\mathrm{SOHO}$ and in particular the SOI. team at the Stanford University for making $S O H O$ MDI data avaiable. Xuyu Wang is supported by the National Basic Research Program of China (2006CB806303).

\section{References}

Aellig, M. R., Holweger, H., Bochsler, P., et al. 1999, in Solar Wind Nine Conference, AIP Conf. Ser., 471, 255

Aellig, M. R., Lazarus, A. J., \& Steinberg, J. T. 2001, Geophys. Res. Lett., 28, 2767

Anders, E., \& Grevesse, N. 1989, Geochim. Cosmochim. Acta, 53, 197

Arge, C. N., \& Mullan, D. J. 1998, Sol. Phys., 182, 293

Bochsler, P. 2007, A\&AR, 14, 1

Bogdan, Y. J., \& Low, B. C. 1986, ApJ, 306, 271

Chen, J. 1996, JGR, 101, 27499

Doschek, G. A. 1983, Sol. Phys., 86, 9

Doschek, G. A., \& Laming, J. M. 2000, ApJ, 539, L71

Feldman, U. 1992, Phys. Scr., 46, 202

Fisk, L. A. 2003, J. Geophys. Res., 108(A4), 1157

Fisk, L. A., \& Zurbuchen, T. H. 2006, J. Geophys. Res., 111, A09115

Fisk, L. A., Schwadron, N. A., \& Zurbuchen, T. H. 1999, J. Geophys. Res., 104, 19765

Geiss, J., et al. 1994, Eos Trans. AGU, 75, \#16, 278

Gopalswamy, N., Shimojo, M., Lu, W., et al. 2003, ApJ, 586, 562

Hénoux, J. C. 1998, Space Sci. Rev., 85, 215

Hildner, E., Gosling, J. T., MacQueen, R. M., et al. 1976, Sol. Phys., 48, 127

Kahler, S. W. 1992, ARA\&A, 30, 113

Kartavykh, Y. Y., Dröge, W., Klecker, B., et al. 2007, ApJ, 671, 947

Kasper, J. C., Stevens, M. L., Lazarus, A. J., et al. 2007, ApJ, 660, 901

Klecker, B., Möbius, E., Popecki, M. A., et al. 2006, Adv. Space Res., 38, 493
Koutchmy, S. 1977, Solar corona, in Illustrated Glossary for Solar and Solar-Terrestrial Physics, ed. A. Bruzek, \& C. J. Durrant (Nowell, Mass.: D. Reidel), 39

Laming, J. M. 2004, ApJ, 614, 1063

Laming, J. M., \& Lepri, S. T. 2007, ApJ, 660, 1642

Levine, R. H. 1982, Sol. Phys., 79, 203

Liewer, P. C., Neugebauer, M., \& Zurbuchen, T. H. 2004, Sol. Phys., 223, 209

Liu, Y., \& Hayashi, K. 2006, ApJ, 640, 1135

Marsch, E., von Steiger, R., \& Bochsler, P. 1995, A\&A, 301, 261

McKenzie, J. F., \& Feldman, U. 1992, ApJ, 389, 764

Meyer, J.-P. 1985, ApJS, 57, 151

Möbius, E., et al. 2003, Proc. 28th Int. Cosmic Ray Conf. (Tsukuba), 6, 3273

Neugebauer, M., Liewer, P. C., Smith, E. J., et al. 2002, J. Geophys. Res., 107, 1488

Parker, E. N. 1958, ApJ, 128, 664

Poduval, B., \& Zhao, X.-P. 2004, J. Geophys. Res., 109(A8), A08102

Reames, D. V. 1999, Space Sci. Rev., 90, 413

Reisenfeld, D. B., Gosling, J. T., Forsyth, R. J., et al. 2003, Geophys. Res. Lett., 30(19), 8031

Schatten, K. H. 1971, Cosmic Electrodyn., 2, 232

Schwadron, N. A., Fisk, L. A., \& Zurbuchen, T. H. 1999, ApJ, 521, 859

Sheeley, N. R. 1996, ApJ, 469, 423

Smith, E. J., \& Balogh, A. 1995, Geophys. Res. Lett., 22, 3317

Suess, S. T., \& Smith, E. J. 1996, Geophys. Res. Lett., 23, 3267

Uzzo, M., Ko, Y.-K., Raymond, J. C., et al. 2003, ApJ, 585, 1062

von Steiger, R., Wimmer Schweingruber, R. F., Geiss, J., et al. 1995, Adv. Space Res., 15,3

Wang, X., Klecker, B., \& Wurz, P. 2008, ApJ, 678, L145

Wang, Y. M., Möbius, E., Popecki, M. A., et al. 1998, ApJ, 508, 899

Widing, K. G., \& Feldman, U. 1992, ApJ, 392, 715

Wurz, P., Möbius, E., Popecki, M. A., et al. 1998, Geophys. Res. Lett., 25, 2557

Wurz, P., Möbius, E., Popecki, M. A., et al. 1999, Phys. Chem. Earth(C), 24(4), 421

Wurz, P., Bochsler, P., Paquette, J. A., \& Ipavich, F. M. 2003a, ApJ, 583, 489

Wurz, P., Wimmer-Schweingruber, R. F., Bochsler, P., et al. 2003b, Solar Wind X Conference, AIP Conf. Proc., 679, 685

Yashiro, S., et al. 2004, J. Geophys. Res., 109, Issue A7, CiteID A07105

Zhao, X.-P., \& Hoeksema, J. T. 1995, J. Geophys. Res., 100, 19

Zhao, X.-P., Hoeksema, J. T., \& Rich, N. B. 2002, Adv. Space Res., 29, 411

Zhou, G.-P., Wang, J. X., \& Cao, Z. L. 2003, A\&A, 397, 1057 